\title{
Color Histogram and DBC Co-Occurrence Matrix for Content Based Image Retrieval
}

\author{
K. Prasanthi Jasmine ${ }^{1}$, P. Rajesh Kumar ${ }^{2}$ \\ ${ }^{1}$ Research Scholar, ${ }^{2}$ Professor \& Head \\ Department of Electronics and Communication Engineering Andhra University, Visakhapatnam Andhra Pradesh, India \\ Email: ${ }^{1}$ jasminekprasanthi@gmail.com, ${ }^{2}$ rajeshauce@gmail.com
}

\begin{abstract}
This paper presents the integration of color histogram and DBC co-occurrence matrix for content based image retrieval. The exit DBC collect the directional edges which are calculated by applying the first-order derivatives in $0^{\circ}, 45^{\circ}, 90^{\circ}$ and $135^{\circ}$ directions. The feature vector length of DBC for a particular direction is 512 which are more for image retrieval. To avoid this problem, we collect the directional edges by excluding the center pixel and further applied the rotation invariant property. Further, we calculated the cooccurrence matrix to form the feature vector. Finally, the HSV color histogram and the DBC co-occurrence matrix are integrated to form the feature database. The retrieval results of the proposed method have been tested by conducting three experiments on Brodatz, MIT VisTex texture databases and Corel-1000 natural database. The results after being investigated show a significant improvement in terms of their evaluation measures as compared to LBP, DBC and other transform domain features.
\end{abstract}

Index Terms - Color, Directional Binary Code, Texture, Pattern Recognition, Feature Extraction, Local Binary Patterns, Image Retrieval.

\section{INTRODUCTION}

CBIR allows searching relevant images to the user's query from an image collection. From last two decades research in CBIR is progressing because of large amounts of digital data archives in scientific, industrial, medical, environmental, educational, entertainment, web image searching and other applications. Analysis and processing of these digital images is difficult so, it is required to have an efficient algorithm. Before 1990 text based image retrieval was being used for this purpose. To reduce the amount of labor in image annotation and different interpretation of images by different persons, in early 1990 CBIR came into the picture. Still there remain some challenging problems that attract the researchers' interest towards CBIR. Generally in CBIR, global visual features related to color, shape, texture, and spatial layout are extracted in the form of color histogram, color coherence, color correlogram, Gabor transform etc. Comprehensive and extensive literature survey on CBIR is presented in [1]-[4].
Swain et al. proposed the concept of color histogram in 1991 and also introduced the histogram intersection distance metric to measure the distance between the histograms of images [5]. Stricker et al. used the first three central moments called mean, standard deviation and skewness of each color for image retrieval [6]. Pass et al. introduced color coherence vec-tor (CCV) [7]. $\mathrm{CCV}$ partitions the each histogram bin into two types, i.e., coherent, if it belongs to a large uniformly colored region or incoherent, if it does not. Huang et al. used a new color feature called color correlogram [8] which characterizes not only the color distributions of pixels, but also spatial correlation of pair of colors. Lu et al. proposed color feature based on vector quantized (VQ) index histograms in the discrete cosine transform (DCT) domain. They computed 12 histograms, four for each color component from 12 DCT-VQ index sequences [9].

Texture is used to specify the roughness or coarseness of object surface and described as a pattern with some kind of regularity. Many researchers have put forward various algorithms for texture analysis, such as the gray co-occurrence matrixes [10], Markov random field (MRF) model [11], simultaneous auto-regressive (SAR)model [12], Wold decomposition model [13], Gabor filtering $[14,15]$ and wavelet decomposition $[16,17]$ and so on. Subrahmanyam et al. [18] combined the color (color histogram) and texture (wavelet transform) features for CBIR. Subrahmanyam et al. proposed correlogram algorithm for image retrieval using wavelets and rotated wavelets (WC+RWC) [19].

Ojala et al. proposed the local binary patterns (LBP) for texture description [20] and these LBPs are converted to rotational invariant for texture classification [21]. pietikainen et al. proposed the rotational invariant texture classification using feature distributions [22]. Ahonen et al. [23] and Zhao et al [24] used the LBP operator facial expression analysis and recognition. Heikkila et al. proposed the background modeling and detection by using LBP [25]. Huang et al. proposed the extended LBP for shape localization [26]. Heikkila et al. used the LBP for interest region description [27]. Li et al. used the combination of Gabor filter and LBP for texture segmentation [28]. Zhang et al. proposed the local derivative pattern for face recognition [29]. They have considered LBP as a nondirectional first order local pattern, which are the binary results of the first-order derivative in images. B. Zhang et al. [30] have proposed 
the directional binary code (DBC) for face recognition. The DBC is encodes the directional edge information in a neighborhood. $\mathrm{C} \mathrm{H}$ Lin et al. [31] combined the color feature, k-mean color histogram (CHKM) and texture features, motif co-occurrence matrix (MCM) and difference between the pixels of a scan pattern (DBPSP). $\mathrm{MCM}$ is the conventional pattern co-occurrence matrix that calculates the probability of the occurrence of same motif between each motif and its adjacent ones in each motif transformed image, and this probability is considered as the attribute of the image. According to the sequence of motifs of scan patterns, DBPSP calculates the difference between pixels and converts it into the probability of occurrence on the entire image. Each pixel color in an image is then replaced by one color in the common color palette that is most similar to color so as to clas-sify all pixels in image into k-cluster, called the CHKM feature.

To improve the retrieval performance in terms of retrieval accuracy, in this paper, we calculated the cooccurrence matrix on DBC patterns. The performance of the proposed method has been tested on Brodatz and MIT VisTex databases for proving the worth of our algorithm. The results after investigation show a significant improvement in terms of their evaluation measures as compared to LBP and DBC.

The organization of the paper as follows: In section 1, a brief review of image retrieval and related work is given. Section 2, 3, 4 and 5 presents a concise review of color histogram, local binary patterns, directional binary code (DBC) and the proposed system framework respectively. Experimental results and discussions are given in section 5. Based on above work conclusions are derived in section 6 .

\section{COLOR Histogram}

In content based Image retrieval, color descriptor has been one of the first choices because if one chooses a proper representation, it can be partially reliable even in presence of changes in lighting, view angle, and scale. In image retrieval, the color histogram is the most commonly used global color feature. It denotes the probability of the intensities of the three color channels.

Typical characterization of color composition is done by color histograms. In 1991 Swain and Ballard [5] proposed the method, called color indexing, which identifies the object using color histogram indexing. The color histogram is obtained by counting the number of times each color occurs in the image array. Histogram is invariant to translation and rotation of the image plane, and change only slowly under change of angle of view [5].

A color histogram $H$ for a given image is defined as a vector

$$
H=\{H[0], H[1], \ldots . ., H[i], \ldots . H[N]\}
$$

Where $i$ represent the color in color histogram and $H[i]$ represent the number of pixels of color $i$ in the image, and
$N$ is the number of bins used in color histogram. For comparing the histogram of different sizes, color histogram should be normalized. The normalized color histogram is given as

$$
H^{\prime}=\frac{H}{p}
$$

where $p$ is the total number of pixels in the image.

In this paper, RGB color space is converted into HSV color space and these are used to calculate the histogram for $\mathrm{H}, \mathrm{S}$ and V color channel.

\section{LOCAL BINARY PATTERNS}

The LBP operator was introduced by Ojala et al. [20] for texture classification. Success in terms of speed (no need to tune any parameters) and performance is reported in many research areas such as texture classification, face recognition, object tracking, bio-medical image retrieval and finger print recognition.

Given a center pixel in the $3 \times 3$ pattern, LBP value is computed by comparing its gray scale value with its neighborhoods based on Eq. (3) and Eq. (4):

$$
\begin{gathered}
L B P_{P, R}=\sum_{i=1}^{P} 2^{(i-1)} \times f\left(I\left(g_{i}\right)-I\left(g_{c}\right)\right) \\
f(x)= \begin{cases}1 & x \geq 0 \\
0 & \text { else }\end{cases}
\end{gathered}
$$

where $I\left(g_{c}\right)$ denotes the gray value of the center pixel, $I\left(g_{i}\right)$ is the gray value of its neighbors, $P$ stands for the number of neighbors and $R$, the radius of the neighborhood.

Fig. 1 shows an example of obtaining an LBP from a given $3 \times 3$ pattern. The histograms of these patterns extract the distribution of edges in an image [20].
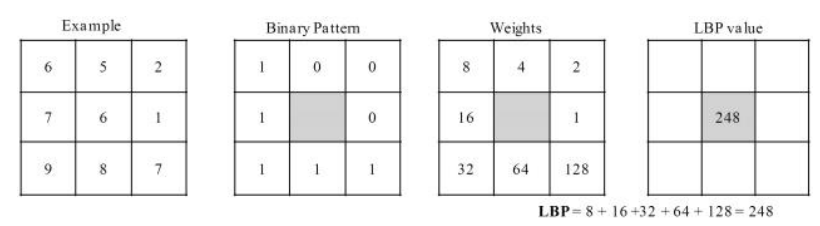

Fig. 1. LBP calculation for $3 \times 3$ pattern

\section{Directional Binary Code}

B. Zhang et al. [30] have proposed the directional binary code (DBC) for face recognition. The DBC is proposed to encode the directional edge information in a neighborhood. Given an image $I$, they denoted its firstorder derivatives along $0^{\circ}, 45^{\circ}, 90^{\circ}$ and $135^{\circ}$ directions as $I_{\alpha, d}^{1}$, where $\alpha=0^{\circ}, 45^{\circ}, 90^{\circ}$ and $135^{\circ}$, and $d$ is the distance between the given point and its neighboring point. For example, in Fig. 2 the distance between the 
center point and its four directional neighbors is 1, i.e. $d=$ 1 in four directions. Let $Z_{i, j}$ be a point in $I$, then the four directional derivatives at $Z_{i, j}$ are

$$
\begin{aligned}
& I_{0^{\circ}, d}^{1}=I\left(Z_{i, j}\right)-I\left(Z_{i, j-d}\right) \\
& I_{45^{\circ}, d}^{1}=I\left(Z_{i, j}\right)-I\left(Z_{i-d, j+d}\right) \\
& I_{90^{\circ}, d}^{1}=I\left(Z_{i, j}\right)-I\left(Z_{i-d, j}\right) \\
& I_{135^{\circ}, d}^{1}=I\left(Z_{i, j}\right)-I\left(Z_{i-d, j-d}\right)
\end{aligned}
$$

A thresholding function, $f\left(I_{\alpha, d}^{1}(Z)\right)$, is applied to the four directional derivatives to output a binary code in the given direction:

$$
f\left(I_{\alpha, d}^{1}(Z)\right)=\left\{\begin{array}{l}
1, \text { if } I_{\alpha, d}^{1}(Z) \geq 0 \\
0, \text { else }
\end{array}\right.
$$

The DBC is defined as:

$$
\left.\operatorname{DBC}\left(I\left(g_{c}\right)\right)\right|_{\alpha}=\left\{I_{\alpha, d}^{1}\left(g_{c}\right), I_{\alpha, d}^{1}\left(g_{1}\right) ; I_{\alpha, d}^{1}\left(g_{2}\right) ; \ldots \ldots \ldots I_{\alpha, d}^{1}\left(g_{8}\right)\right\}
$$

Fig. 2 illustrates the DBC calculation in $0^{\circ}$ direction.

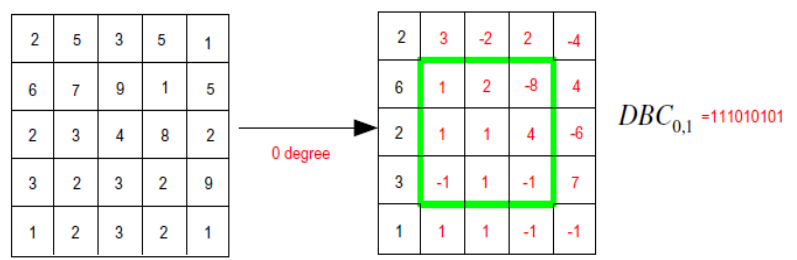

Fig. 2. An example of DBC calculation in $0^{\circ}$ direction.

From Eq. (7), it is clear that the DBC contains 9 binary bits. Hence the feature vector length is 512. To solve this feature vector length problem, we coded the DBC by excluding the center pixel edge value as shown in Eq. (8).

$$
\left.\operatorname{DBC}\left(I\left(g_{c}\right)\right)\right|_{\alpha}=\left\{I_{\alpha, d}^{1}\left(g_{1}\right) ; I_{\alpha, d}^{1}\left(g_{2}\right) ; \ldots \ldots \ldots I_{\alpha, d}^{1}\left(g_{8}\right)\right\}
$$

The uniform pattern refers to the uniform appearance pattern which has limited discontinuities in the circular binary presentation. In this paper, the pattern which has less than or equal to two discontinuities in the circular binary presentation is considered as the uniform pattern and remaining patterns considered as non-uniform patterns.

The distinct values for given query image is $P(P-1)+3$ by using uniform patterns [31]. But these features are not rotational invariant. The rotational invariant patterns $\left(D B C_{P, R}^{r i u 2}\right)$ can be constructed by adding all eight patterns in the each row [31]. The distinct values for a given query image is $P+2$ by using rotational invariant patterns $\left(D B C_{P, R}^{\text {riu2 }}\right)$. After DBC calculation, the co-occurence matrix is calculated for feature vector generation.

\subsection{Calligraphic image pre-processing}

In this paper, the co-occurrence matrix is used to represent the traversal of adjacent pattern difference in an DBC image.

The co-occurrence matrix calculates the distribution within the two-dimensional pattern matrix $P_{i}\left[N_{x}, N_{y}\right]$. That is, it takes into account the probability of the cooccurrence between the two DBC pattern respectively corresponding to $(x, y)$ and it's adjacent $(x+d x, y+d y)$. This probability is then the attribute of image pattern variation used in this paper. The coordinate that distances from ( $\mathrm{x}, \mathrm{y})$ on the $\mathrm{x}$ axis in $\mathrm{dx}$ and on $\mathrm{y}$ axis in $d y$, then the total number of co-occurring pattern pairs $(u, v)$ (where $u=0,1, \ldots, 6$ and $v=0,1, \ldots, 6$ ) is determined by

$$
M_{i}(u, v)=M_{i}(u, v \mid d x, d y)=M_{i}\left(P_{i}[x, y], P_{i}[x+d x, y+d y]\right)
$$

where $P_{i}[x, y]=\mathrm{u}, P_{i}[x+d x, y+d y]=v, 1 \leq i \leq 4,1 \leq x \leq N_{x}$, $1 \leq y \leq N_{y}, 1 \leq x+d x \leq N_{x}$, and $1 \leq y+d y \leq N_{y}$.

\section{DiRECTIONAL BINARY CODE}

In this paper, we proposed the new technique by integrating the co-occurrence matrix on rotational invariant DBC subimages and color histogram. Finally, feature vector is constructed by concatenating the features collected using three directional co-occurrence matrix on four directional DBC. Fig. 3 illustrates the flowchart of the proposed system framework and algorithm for the same is given bellow.

\section{Algorithm:}

Input: Image; Output: Retrieval results.

1. Load the input image.

2. Convert RGB image to HSV.

3. Quantize the HSV spaces.

4. Construct the histograms.

5. Convert RGB image into a grayscale image.

6. Perform the first-order derivative in $0^{\circ}, 45^{\circ}, 90^{\circ}$ and $135^{\circ}$ directions.

7. Calculate the DBC and make them into rotational invariant.

8. Calculate the co-occurrence matrix.

9. Form the feature vector by concatenating all cooccurrence matrixes (step 8) and color histigrams (step 4).

10. Calculate the best matches using Eq. (10).

11. Retrieve the number of top matches.

\subsection{Similarity Measurement}

In the presented work $d_{1}$ similarity distance metric ise used as shown below:

$$
D(Q, T)=\sum_{i=1}^{L g}\left|\frac{f_{T, i}-f_{Q, i}}{1+f_{T, i}+f_{Q, i}}\right|
$$


where $Q$ is query image, $L g$ is feature vector length, $T$ is image in database; $f_{I, i}$ is $i^{\text {th }}$ feature of image $I$ in the database, $f_{Q, i}$ is $i^{\text {th }}$ feature of query image $Q$.

\section{EXPERIMENTAL RESULTS AND DISCUSSIONS}

For the work reported in this paper, retrieval tests are conducted on three benchmark databases and results are presented in the following subsections.

\subsection{Experiment \#1}

In this experiment, the Brodatz database is used which consists of 116 different textures comprising of 109 textures from Brodatz texture photographic album [32], seven textures from USC database [33]. The size of each texture is $512 \times 512$ and is further divided into sixteen $128 \times 128$ non-overlapping sub-images, thus creating a database of $1856(116 \times 16)$ images.

The performance of the proposed method is measured in terms of average retrieval precision (ARP) and average retrieval rate by following equations.

$$
\begin{gathered}
\text { Precision }(P)=\frac{\text { No.of Relevant Images Retrieved }}{\text { Total No.of Images Retrieved }} \times 100 \\
\text { Group Precision }(G P)=\frac{1}{N_{1}} \sum_{i=1}^{N_{1}} P \\
\text { Average Retrieval Precision }(A R P)=\frac{1}{\Gamma_{1}} \sum_{j=1}^{\Gamma_{1}} G P
\end{gathered}
$$

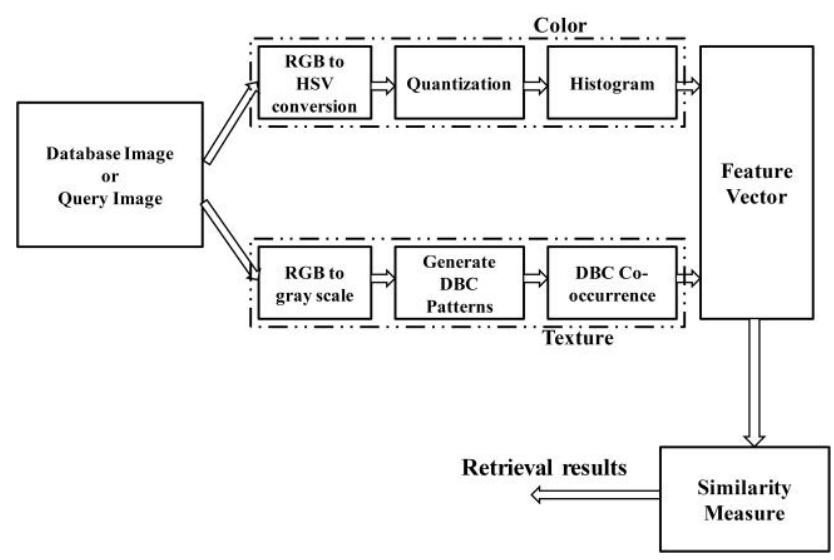

Fig. 3: Proposed system framework

$$
\begin{array}{r}
\operatorname{Recall}(R)=\frac{\text { Number of relevant images retrieved }}{\text { Total Number of relevant images }} \\
\text { Group Recall }(G R)=\frac{1}{N_{1}} \sum_{i=1}^{N_{1}} R \\
\text { Average Retrieval Rate }(A R R)=\frac{1}{\Gamma_{1}} \sum_{j=1}^{\Gamma_{1}} G R
\end{array}
$$

where $N_{1}$ is number of relevant images and $\Gamma_{1}$ is number of groups.

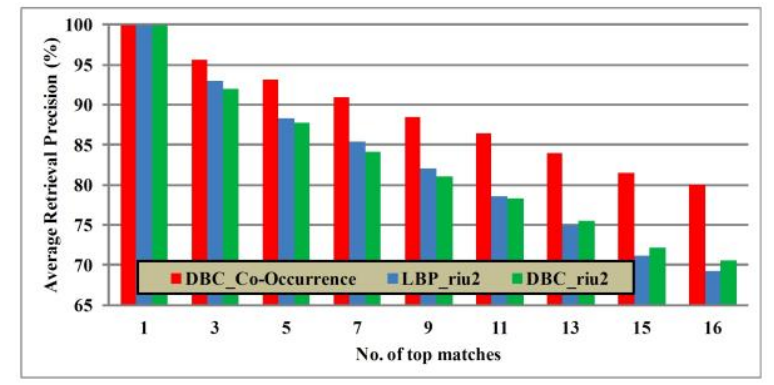

(a)

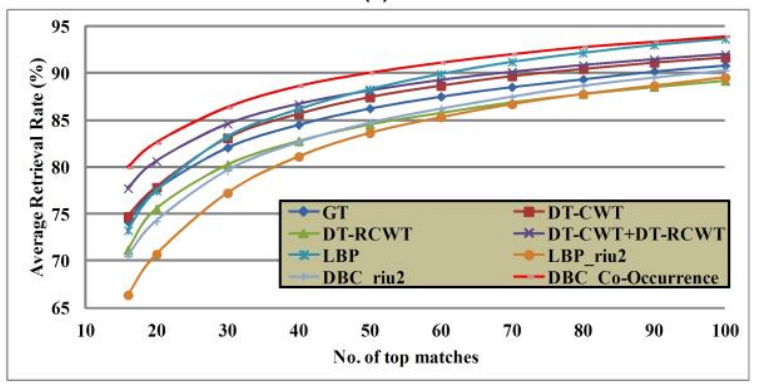

(b)

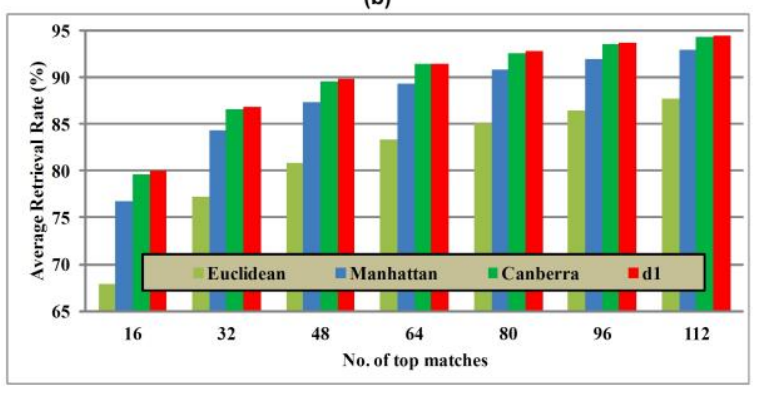

(c)

Fig. 4. Comparison of proposed method with LBP, DBC and other exiting transform domain methods in terms of: (a) average retrieval precision, (b) average retrieval rate and (c) performance of proposed method using various distance measures.

Table 1 \& Fig. 4 (a) and Table 2 \& Fig. 4 (b) summarize the retrieval results of the proposed method (DBC_Co-occurence), LBP, DBC and other existing transform domain methods in terms of average retrieval precision and rate respectively. From Tables $1 \& 2$ and Fig. 4 (a) \& (b), it is clear that the proposed method showing better performance compared to LBP, DBC and other existing methods in terms of average retrieval precision and rate. The performance of the proposed method also tested with different distance measures and observed the $d_{1}$ distance outperforming the other distances as showing Table 4 and Fig. 4 (c).

\subsection{Experiment \#2}

The MIT VisTex database is used in our experiment which consists of 40 different textures [34]. The size of each texture is $512 \times 512$. Each $512 \times 512$ image is divided into sixteen $128 \times 128$ non-overlapping subimages, thus creating a database of $640(40 \times 16)$ images. The performance of the proposed method is measured in terms of average retrieval rate (ARR) is given by Eq. (15).

$$
A R R=\frac{\text { No.of Relevant Images Retrieved }}{\text { Total No.of Relevant Images in Database }} \times 100
$$




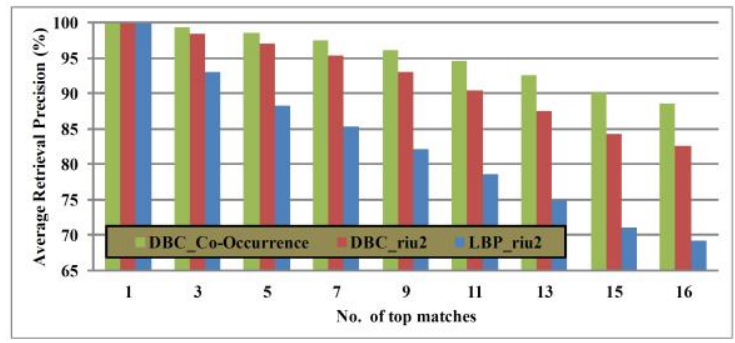

(a)

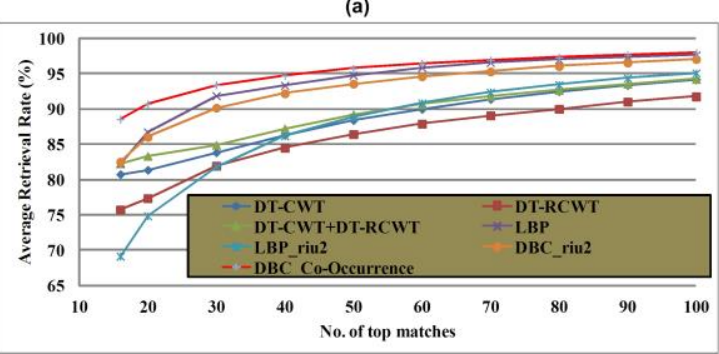

(b)

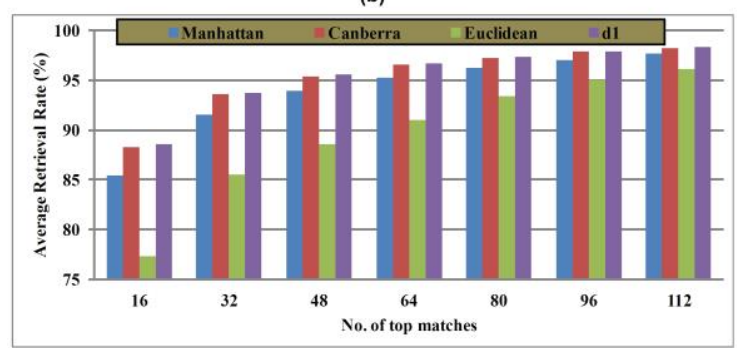

(c)

Fig. 5. Comparison of proposed method with LBP, DBC and other exiting transform domain methods in terms of: (a) average retrieval precision, (b) average retrieval rate and (c) performance of proposed method using various distance measures.
This database is used to compare the performance of the proposed method (DBC_Co-Occurence) with LBP, DBC and other existing methods. Fig. 5 (a) \& (b) illustrate the retrieval results of proposed method and other existing methods in terms of average retrieval precision and average retrieval rate respectively. From Fig. 5 (a) \& (b), it is evident that the proposed method is outperforming the other existing methods. The results of the proposed method are also compared with the different distance measures as shown in Fig. 5 (c). From Fig. 5 (c), it is found that the $d_{1}$ distance is outperforming the other distances.

Table 1. Retrieval Results Of All Techniques In Terms Of Average Retrieval Precision On Brodatz Database

\begin{tabular}{|l|l|l|l|l|l|l|l|l|l|}
\hline Method & \multicolumn{9}{|l|}{ Number of top matches } \\
& $\mathbf{1}$ & $\mathbf{3}$ & $\mathbf{5}$ & $\mathbf{7}$ & $\mathbf{9}$ & $\mathbf{1 1}$ & $\mathbf{1 3}$ & $\mathbf{1 5}$ & $\mathbf{1 6}$ \\
\hline LBP_riu2 & 100 & 93.02 & 88.31 & 85.37 & 82.08 & 78.50 & 74.92 & 71.08 & 69.16 \\
\hline DBC_riu2 & 100 & 91.95 & 87.69 & 84.10 & 81.09 & 78.31 & 75.43 & 72.20 & 70.51 \\
\hline DBC_Co-Occurrence & 100 & 95.61 & 93.09 & 90.86 & 88.50 & 86.35 & 84.00 & 81.45 & 79.94 \\
\hline
\end{tabular}

Table 2. Retrieval results of all techniques in terms of average retrieval Rate on Brodatz database

\begin{tabular}{|c|c|c|c|c|c|c|c|c|c|c|}
\hline Method & \multicolumn{10}{|c|}{ Number of top matches } \\
\hline & $\mathbf{1 6}$ & $\mathbf{2 0}$ & $\mathbf{3 0}$ & $\mathbf{4 0}$ & $\mathbf{5 0}$ & $\mathbf{6 0}$ & $\mathbf{7 0}$ & $\mathbf{8 0}$ & $\mathbf{9 0}$ & $\mathbf{1 0 0}$ \\
\hline GT & 74.1 & 77.5 & 82.0 & 84.5 & 86.2 & 87.5 & 88.5 & 89.3 & 90.2 & 90.8 \\
\hline DT-CWT & 74.7 & 77.85 & 83.1 & 85.67 & 87.46 & 88.69 & 89.71 & 90.48 & 91.16 & 91.7 \\
\hline DT-RCWT & 71.1 & 75.56 & 80.25 & 82.79 & 84.56 & 85.79 & 86.93 & 87.81 & 88.56 & 89.23 \\
\hline DT-CWT+DT-RCWT & 77.7 & 80.63 & 84.59 & 86.71 & 88.14 & 89.27 & 90.14 & 90.86 & 91.47 & 92.02 \\
\hline LBP & 73.2 & 77.47 & 83.23 & 86.16 & 88.31 & 89.92 & 91.21 & 92.184 & 93.03 & 93.67 \\
\hline LBP_riu2 & 66.3 & 70.72 & 77.25 & 81.13 & 83.64 & 85.32 & 86.72 & 87.79 & 88.69 & 89.56 \\
\hline DBC_riu2 & 70.5 & 74.23 & 79.63 & 82.63 & 84.70 & 86.21 & 87.48 & 88.66 & 89.52 & 90.29 \\
\hline DBC_Co-Occurrence & 79.9 & 82.58 & 86.36 & 88.63 & 90.04 & 91.13 & 92.04 & 92.81 & 93.36 & 93.93 \\
\hline
\end{tabular}


Table 3. Results Of Proposed Method using Various Distance Measures In Terms Of Average Retrieval Rate On Brodatz database

\begin{tabular}{|c|c|c|c|c|c|c|c|}
\hline \multirow[t]{2}{*}{ Method } & \multicolumn{7}{|c|}{ Number of top matches } \\
\hline & 16 & 32 & 48 & 64 & 80 & 96 & 112 \\
\hline Manhattan & 76.73 & 84.30 & 87.36 & 89.30 & 90.83 & 91.98 & 92.91 \\
\hline Canberra & 79.63 & 86.60 & 89.62 & 91.41 & 92.60 & 93.57 & 94.38 \\
\hline Euclidean & 67.87 & 77.20 & 80.85 & 83.34 & 85.08 & 86.53 & 87.75 \\
\hline$d_{1}$ & 79.94 & 86.89 & 89.85 & 91.49 & 92.81 & 93.71 & 94.48 \\
\hline
\end{tabular}

Table 4. Results of various techniques in terms of precision (\%) Corel-1000 database

\begin{tabular}{|l|c|c|c|c|}
\hline \multirow{2}{*}{ Category } & \multicolumn{2}{|l|}{ Precision $(\boldsymbol{n}=\mathbf{2 0})$} & \multicolumn{2}{l|}{} \\
\cline { 2 - 5 } & $\mathbf{C ~ H}$ Lin et al & CC & DBC & DBC Co-occurrence +Color Hist \\
\hline Africans & 68.3 & 80.4 & 58.9 & 70.8 \\
\hline Beaches & 54 & 41.25 & 56.3 & 53.8 \\
\hline Buildings & 56.2 & 55.65 & 61.4 & 74.8 \\
\hline Buses & 88.8 & 76.7 & 96.5 & 98.6 \\
\hline Dinosaurs & 99.3 & 99 & 98.6 & 98.2 \\
\hline Elephants & 65.8 & 56.2 & 43.7 & 52.7 \\
\hline Flowers & 89.1 & 92.9 & 90.8 & 92.1 \\
\hline Horses & 80.3 & 76.5 & 70.6 & 83.5 \\
\hline Mountains & 52.2 & 33.7 & 38.6 & 37.3 \\
\hline Food & 73.3 & 70.6 & 73.5 & 87.2 \\
\hline Total & $\mathbf{7 2 . 7}$ & $\mathbf{6 8 . 2}$ & $\mathbf{6 8 . 9}$ & $\mathbf{7 4 . 9}$ \\
\hline
\end{tabular}

n-No. top matches considered

Table 5. Results of various techniques in terms of recall (\%) Corel-1000 database

\begin{tabular}{|l|c|c|c|}
\hline \multirow{2}{*}{ Category } & \multicolumn{2}{|l|}{ Recall $(\boldsymbol{n}=\mathbf{1 0 0})$} & DBC \\
\cline { 2 - 4 } & CC & 33.5 & 41.9 \\
\hline Africans & 46.29 & 36.1 & 37.9 \\
\hline Beaches & 25.29 & 35.6 & 42.1 \\
\hline Buildings & 35.01 & 64.1 & 72.6 \\
\hline Buses & 60.97 & 84.0 & 89.1 \\
\hline Dinosaurs & 89.59 & 29.7 & 33.5 \\
\hline Elephants & 34.14 & 70.6 & 73.8 \\
\hline Flowers & 77.69 & 37.7 & 49.5 \\
\hline Horses & 36.13 & 23.3 & 25.0 \\
\hline Mountains & 21.02 & 50.2 & 56.2 \\
\hline Food & 39.27 & $\mathbf{4 6 . 5}$ & $\mathbf{5 2 . 2}$ \\
\hline Total & $\mathbf{4 6 . 5}$ & & \\
\hline
\end{tabular}

$n-$ No. top matches considered

\subsection{Experiment \#3}

This experiment is conducted to prove the worth of proposed algorithm which integrates the color histogram and DBC co-occurrence matrix for natural image retrieval.

In this experiment Corel-1000 natural image database is used. Corel database [35] contains large amount of images of various contents ranging from animals and outdoor sports to natural images. These images are preclassified into different categories of size 100 by domain professionals. Some researchers think that Corel database meets all the requirements to evaluate an image retrieval system, because of its large size and heterogeneous content. In this paper, we collected the database DB1 contains 1000 images of 10 different categories (groups $G)$. Ten categories are provided in the database namely Africans, beaches, buildings, buses, dinosaurs, elephants, flowers, horses, mountains and food. Each category has 100 images $\left(N_{G}=100\right)$ and these have either $256 \times 384$ or $384 \times 256$ sizes. The performance of the proposed 
method is measured in terms of ARP and ARR.

Table 4 and 5 summarizes the retrieval results of the proposed method (CLBP), LBP and other previously available methods (C $\mathrm{H}$ Lin et al. [31], and color correlogram (CC) [8]) in terms of average retrieval precision and recall respectively. From Table 4 and Table 5 , it is clear that the proposed method showing better performance compared to DBC and other previously available methods in terms of average retrieval precision and recall on Corel-1000 natural database.

\section{CONCLUSIONS}

A new image indexing and retrieval algorithm which integrates the color histogram and DBC co-occurrence matrix is proposed in this paper by calculating the cooccurrence matrix on DBC subimages. The experiments have been carried out on Corel-1000, Brodatz and MIT VisTex databases for proving the worth of our algorithm. The results after being investigated show a significant improvement in terms of their evaluation measures as compared to LBP, DBC and other transform domain features.

\section{REFERENCES}

[1] Y. Rui and T. S. Huang, Image retrieval: Current techniques, promising directions and open issues, J.. Vis. Commun. Image Represent., 10 (1999) 39-62.

[2] A. W.M. Smeulders, M. Worring, S. Santini, A. Gupta, and R. Jain, Content-based image retrieval at the end of the early years, IEEE Trans. Pattern Anal. Mach. Intell., 22 (12) 1349-1380, 2000.

[3] M. Kokare, B. N. Chatterji, P. K. Biswas, A survey on current content based image retrieval methods, IETE J. Res., 48 (3\&4) 261-271, 2002.

[4] Ying Liu, Dengsheng Zhang, Guojun Lu, Wei-Ying Ma, Asurvey of content-based image retrieval with high-level semantics, Elsevier J. Pattern Recognition, 40, 262-282, 2007.

[5] M. J. Swain and D. H. Ballar, Indexing via color histograms, Proc. 3rd Int. Conf. Computer Vision, Rochester Univ., NY, (1991) 11-32.

[6] M. Stricker and M. Oreng, Similarity of color images, Proc. SPIE, Storage and Retrieval for Image and Video Databases, (1995) 381-392.

[7] G. Pass, R. Zabih, and J. Miller, Comparing images using color coherence vectors, Proc. 4th ACM Multimedia Conf., Boston, Massachusetts, US, (1997) 65-73.

[8] J. Huang, S. R. Kumar, and M. Mitra, Combining supervised learning with color correlograms for contentbased image retrieval, Proc. 5th ACM Multimedia Conf., (1997) 325-334.

[9] Z. M. Lu and H. Burkhardt, Colour image retrieval based on DCT domain vector quantization index histograms, J. Electron. Lett., 41 (17) (2005) 29-30.

[10] R.M. Haralick, K. Shangmugam, I. Dinstein, Textural feature for image classification, IEEE Trans. Syst. Man Cybern. SMC-3 (6) (1973) 610-621.

[11] G. Cross, A. Jain, Markov random field texture models, IEEE Trans. Pattern Anal. Mach. Intell. 5 (1) (1983) 25 39.

[12] J. Mao, A. Jain, Texture classification and segmentation using multi-resolution simultaneous autoregressive models, Pattern Recognition 25 (2) (1992) 173-188.

[13] F. Liu, R. Picard, Periodicity, directionality, and randomness: wold features for image modeling and retrieval, IEEE Trans. Pattern Anal. Mach. Intell. 18 (7) (1996) 722-733.

[14] B.S. Manjunath, W.Y. Ma, Texture features for browsing and retrieval of image data, IEEE Trans. Pattern Anal. Mach. Intell. 18 (8) (1996) 837-842.

[15] J. Han, K.-K. Ma, Rotation-invariant and scale-invariant Gabor features for texture image retrieval, Image Vision Comput. 25 (2007) 1474-1481.

[16] T. Chang, C.C. Jay Kuo, Texture analysis and classification with tree-structured wavelet transform, IEEE Trans. Image Process. 2 (4) (1993) 429-441.

[17] A. Laine, J. Fan, Texture classification by wavelet packet signatures, IEEE Trans. Pattern Anal. Mach. Intell. 11 (15) (1993) 1186-1191.

[18] M. Subrahmanyam, A. B. Gonde and R. P. Maheshwari, Color and Texture Features for Image Indexing and Retrieval, IEEE Int. Advance Computing Conf., Patial, India, (2009) 1411-1416.

[19] Subrahmanyam Murala, R. P. Maheshwari, R. Balasubramanian, A Correlogram Algorithm for Image Indexing and Retrieval Using Wavelet and Rotated Wavelet Filters, Int. J. Signal and Imaging Systems Engineering.

[20] T. Ojala, M. Pietikainen, D. Harwood, A comparative sudy of texture measures with classification based on feature distributions, Elsevier J. Pattern Recognition, 29 (1): 51-59, 1996.

[21] T. Ojala, M. Pietikainen, T. Maenpaa, Multiresolution gray-scale and rotation invariant texture classification with local binary patterns, IEEE Trans. Pattern Anal. Mach. Intell., 24 (7): 971-987, 2002.

[22] M. Pietikainen, T. Ojala, T. Scruggs, K. W. Bowyer, C. Jin, K. Hoffman, J. Marques, M. Jacsik, W. Worek, Overview of the face recognition using feature distributions, Elsevier J. Pattern Recognition, 33 (1): 4352,2000

[23] T. Ahonen, A. Hadid, M. Pietikainen, Face description with local binary patterns: Applications to face recognition, IEEE Trans. Pattern Anal. Mach. Intell., 28 (12): 2037-2041, 2006.

[24] G. Zhao, M. Pietikainen, Dynamic texture recognition using local binary patterns with an application to facial expressions, IEEE Trans. Pattern Anal. Mach. Intell., 29 (6): 915-928, 2007.

[25] M. Heikkil;a, M. Pietikainen, A texture based method for modeling the background and detecting moving objects, IEEE Trans. Pattern Anal. Mach. Intell., 28 (4): 657-662, 2006.

[26] X. Huang, S. Z. Li, Y. Wang, Shape localization based on statistical method using extended local binary patterns, Proc. Inter. Conf. Image and Graphics, 184-187, 2004.

[27] M. Heikkila, M. Pietikainen, C. Schmid, Description of interest regions with local binary patterns, Elsevie J. Pattern recognition, 42: 425-436, 2009.

[28] M. Li, R. C. Staunton, Optimum Gabor filter design and local binary patterns for texture segmentation, Elsevie J. Pattern recognition, 29: 664-672, 2008.

[29] B. Zhang, Y. Gao, S. Zhao, J. Liu, Local derivative pattern versus local binary pattern: Face recognition with higher-order local pattern descriptor, IEEE Trans. Image Proc., 19 (2): 533-544, 2010.

[30] B. Zhang, L. Zhang, D. Zhang, L. Shen, Directional binary code with application to PolyU near-infrared face database, Pattern Recognition Letters 31 (2010) 2337 - 
2344.

[31] A. Abdullah, R. C. Veltkamp and M. A. Wiering, Fixed Partitioning and salient points with MPEG-7 cluster correlogram for image categorization, Pattern Recognition, 43, (2010) 650-662.

[32] P. Brodatz, Textures: A Photographic Album for Artists and Designers, New York: Dover, 1996.

[33] University of Southern California, Signal and Image Processing Institute, Rotated Textures. [Online]. Available: http://sipi.usc.edu/database/.

[34] MIT Vision and Modeling Group, Vision Texture. [Online]. Available: http://vismod.www.media.mit.edu.

[35] Corel-1000 natural image database. [Online]. Available: http://wang.ist.psu.edu/docs/related.shtml.

\section{Authors' Profiles}

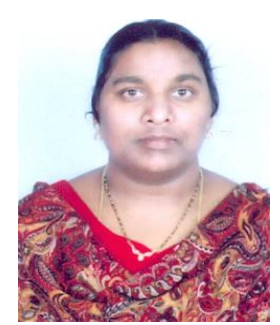

K. prasanthi Jasmine, received her B.Tech in Electronics \& Communication Engineering and M.Tech in Digital Systems from Regional Engineering College( Now NIT), Warangal, Andhra Pradesh, and Osmania University College of Engineering, Osmania University, Andhra Pradesh, India in the years 2000 and 2003 respectively. Currently, she is pursuing Ph.D from Andhra University, A.P, India. Her major fields of interest is Image Retrieval, Digital image Processing and Pattern Recognition.

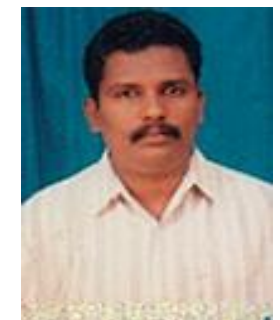

P. Rajesh Kumar received his M.Tech, $\mathrm{Ph} . \mathrm{D}$ degrees from Andhra University, Vishakhapatnam, India. He is currently working as professor, Department of Electronics \& Communication Engineering, Andhra University College of Engineering, Visakhapatnam, Andhra Pradesh. He is also Assistant Principal of Andhra University college of Engineering, Visakhapatnam, Andhra Pradesh. He has twenty years experience of teaching undergraduate and postgraduate students and guided a number of post graduate thesis. He has published twenty research papers in national and international journals and conferences. Presently he is guiding twelve Research scholars. His research interests are digital signal and image processing, computational intelligence, human computer interaction, radar signal processing.

How to cite this paper: K. Prasanthi Jasmine, P. Rajesh Kumar,"Color Histogram and DBC Co-Occurrence Matrix for Content Based Image Retrieval", IJIEEB, vol.6, no.6, pp.47-54, 2014. DOI: 10.5815/ijieeb.2014.06.06 